\title{
Die anderen Dinge der Pädagogik. Zum Umgang mit alltäglichen Gegenständen in Kinderkrippen
}

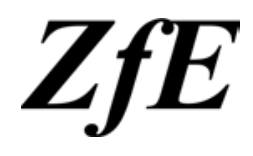

\author{
Sascha Neumann
}

Zusammenfassung: Dinge im Sinne stofflich erfahrbarer Entitäten nehmen historisch wie gegenwärtig in der Diskussion über Programm und Praxis der Frühpädagogik eine herausragende Stellung ein. Die Dominanz des Dinglichen in der Frühpädagogik beruht bis heute auf der Annahme von der Bildungs- und Entwicklungsbedeutsamkeit dinglich-materiell vermittelter Erfahrungen. Mit dieser Annahme wird unterstellt, der Umgang mit den Dingen in der frühpädagogischen Praxis sei vor allem durch die spezifischen Bildungs- und Entwicklungsbedürfnisse von Kindern bestimmt. Offen bleibt dabei, wie es um jene Objekte steht, denen nicht ohne weiteres und von vorne herein eine pädagogische Relevanz zugetraut wird. Der Beitrag knüpft an dieser Frage an und stellt die angestammte frühpädagogische Sicht der Dinge empirisch auf die Probe. Anhand von ethnographischen Beobachtungen zum Gebrauch alltäglicher Gegenstände in Kinderkrippen rekonstruiert er exemplarisch, wie diese Gegenstände für die Selbstbeobachtung der Praxis als ,pädagogisch` gerade insofern eine Bedeutung gewinnen, als sie es gestatten - territorial wie sozial - Kinder als sich verändernde Personen zur Darstellung zu bringen.

Schlüsselwörter: Ethnographie $\cdot$ Frühpädagogik $\cdot$ Materialität $\cdot$ Pädagogisches $\cdot$ Kind

\section{The other things in education. How everyday objects are handled in crèches}

\begin{abstract}
Things in terms of material entities take historically as well as currently a prominent role in the discussion on programs and practices in early childhood education. The dominance of the tangible in early childhood education is based on the assumption of the educational and developmental significance of material experiences in the early ages. Regardless of the validity of this assumption, the pedagogical reflection upon the special role of things also suggests that the overwhelming significance of the tangible in early childhood education is founded solely on the special educational and developmental needs of children. The paper is interrogating this underlying claim by confronting it with empirical insights as they can be obtained from the ethnography of pedagogical practices in early childhood education. This is done by analyzing the use of different everyday pieces in educational practices. The analysis illustrates how these things function to represent children-socially and territorially-as persons in change and thereby gain the status of a medium for proving the everyday practices as something pedagogical.
\end{abstract}

(C) Springer Fachmedien Wiesbaden 2013

Prof. Dr. S. Neumann $(\bowtie)$

Departement Erziehungswissenschaften, Université de Fribourg/Universität Freiburg $(\mathrm{CH})$,

Rue P.-A. Faucigny 2, 1700 Fribourg, Schweiz

E-Mail: sascha.neumann@unifr.ch 
Keywords: Ethnography $\cdot$ Early childhood education $\cdot$ Materiality $\cdot$ Pedagogy $\cdot$ Child

\section{Dinge in der Pädagogik und die Pädagogik der Dinge - eine Einleitung}

Dass es die Pädagogik nicht nur mit Personen, sondern immer auch mit Dingen als nicht belebten, gleichwohl aber stofflich erfahrbaren Entitäten zu tun hat, gehört im Grunde zum kollektiven Wissen all derjenigen, die je mit ihren Institutionen in Berührung gekommen sind. Was wäre die Schule ohne die Tafel, der Kindergarten ohne sein Arsenal an Kuscheltieren, Bällen, Puppen und Bilderbüchern, was der Nähkurs in der Erwachsenenbildung ohne Nadel, Stoff und Faden? Umso überraschender ist es, dass die Erziehungswissenschaft lange Zeit - sieht man einmal von der pädagogischen Anthropologie und vereinzelten Bezugnahmen in der Bildungstheorie und erziehungswissenschaftlichen Historiographie ab (vgl. Langeveld 1955; Mollenhauer 1987; Pazzini 1983; MeyerDrawe 1999; Parmentier 2001) - kaum einmal ein besonderes Interesse für die materielle Dimension ihres Forschungsgegenstandes gezeigt hat (vgl. Nohl 2011, S. 8; Oelkers 2012; Rieger-Ladich 2009). In jüngster Zeit jedoch scheint sich dies allmählich zu ändern. So hat sich inzwischen auch innerhalb der Erziehungswissenschaft eine beachtliche Sensibilität für den epistemischen Stellenwert herausgebildet, den die Analyse des Umgangs mit konkreten Objekten für das Verständnis und die Analyse der Erziehungs- und Bildungswirklichkeit einnehmen könnte (vgl. etwa: Nohl 2011; Casale et al. 2012). Dieser Wandel ist vor allem inspiriert durch Entwicklungen in der Soziologie und Sozialphilosophie, wie etwa der prominent von Bruno Latour vertretenen Akteur-Netwerk-Theorie (ANT) (vgl. Latour 2007) oder Theodore R. Schatzkis Variante einer Theorie sozialer Praktiken (vgl. Schatzki 1996, 2001), aber auch durch die insgesamt wachsende „Konjunktur der Aufmerksamkeit“ für das Materielle in den Sozial- und Kulturwissenschaften (König 2012). Davon versuchen neben vereinzelten theoretisch-systematischen Reflexionen vor allem empirische Arbeiten zu profitieren, die dem Spektrum qualitativer Untersuchungen und im engeren Sinne der ethnographischen bzw. videographischen Forschung zuzuordnen sind (vgl. hierzu etwa Breidenstein 2006; Sørensen 2009; Wiesemann 2009; Bollig et al. 2012; Eßer 2012; Fetzer 2012; Neumann 2012a; Rabenstein und Wieneke 2012; Röhl 2012).

Die jüngste Entwicklung hat zwar die materielle Dimension der pädagogischen Wirklichkeit wieder verstärkt in den Horizont der Aufmerksamkeit gerückt, dennoch ist das Interesse an den Dingen noch ein eher fokussiertes und noch nicht derart grundlegend ausgeprägt, dass damit immer auch schon die Frage adressiert wäre, inwiefern die Dinge als solche sowie ein bestimmter Umgang mit ihnen für die Pädagogizität einer sich selbst als pädagogisch beobachtenden Vollzugswirklichkeit tatsächlich konstitutiv sind. Dies gilt vor allem in empirischer Hinsicht und zeigt sich dabei auf zweierlei Weise (vgl. hierzu Neumann 2012a, S. 172). Entweder wird diese Frage ganz gemieden, um nicht den angestammten normativen Perspektiven über das Pädagogische auf den Leim zu gehen (Fritzsche et al. 2011). Oder aber die Analysen und Beobachtungen konzentrieren sich auf die Dinge des ,Wissens' oder ,Lernens', also jene Materialitäten, die - wie etwa didaktische Objekte des Schulunterrichts - schon qua Herstellung einen pädagogischen Charakter in sich zu tragen scheinen (vgl. hierzu etwa Fetzer 2012; Rabenstein und Wieneke 2012; 
Sørensen 2009; Wiesemann 2009). Insofern gibt es zwar eine zunehmende Auseinandersetzung mit den Dingen der Pädagogik, gleichwohl ist die Empirie einer „Pädagogik der Dinge“ (Nohl 2011) bislang kaum mehr als in Ansätzen erschlossen worden.

Anders als der Erziehungswissenschaft insgesamt darf man der Pädagogik der frühen Kindheit durchaus ein „,inniges“ Verhältnis zu den Dingen unterstellen. Historisch wie gegenwärtig spielen sie eine prominente Rolle in der Diskussion über Programm und Didaktik der Frühpädagogik. Schon Rousseaus „Emile“ sollte sich an ihrer Widerständigkeit bilden und durch ihre „Macht“ gleichsam ,gefügig“ und ,gehorsam“ werden (Rousseau 1971, S.71). Bekanntermaßen wurden die Dinge fortan auch in den zentralen Lehrgebäuden der Frühpädagogik als die wichtigsten Komplizen bei der Verwirklichung ihrer Ansprüche gehandelt. Dies gilt nicht nur für Fröbels „Spielgaben“ (vgl. hierzu Hewitt 2001, Prochner 2011), sondern auch für die „,vorbereitete Umgebung“ Maria Montessoris oder den Raum als sogenannten „dritten Erzieher“, wie ihn die Reggiopädagogik kennt, um nur einige Beispiele zu nennen. Das frühpädagogische Interesse an den Dingen speist sich dabei aus der Überzeugung, dass den materiell vermittelten Erfahrungen eine besondere Bedeutung beim Verlauf, Vollzug und der Gestaltung von Lern-, Entwicklungs- und Bildungsprozessen eingeräumt werden muss (vgl. Stieve 2008, 2011). Insofern ist die frühpädagogische Rede von den „Dingen“ kaum von einer impliziten pädagogischen Programmatik des Umgangs mit ihnen zu trennen. Dies erklärt womöglich auch, warum sie lange Zeit empirisch kaum von Interesse gewesen sind, war doch alles, was sich über sie zu wissen lohnte, bereits in den wohlmeinenden Intentionen des pädagogisch ambitionierten Blicks vorweg genommen (Stieve 2008, S.43, 2011, S. 126-127). Nicht zuletzt suggeriert diese enge Bindung der Perspektive auf die Dinge an die Intentionen der frühpädagogischen Praxis aber auch, die Materialität dieser Praxis begründe sich allein von den spezifischen Bildungs- und Entwicklungsbedürfnissen von Kindern her. Was aber, so kann man fragen, ist mit all jenen Dingen in den Settings öffentlich veranstalteter Kleinkinderziehung, die sich nicht ohne weiteres und von vorne herein in ihrer Bildungs- und Entwicklungsbedeutsamkeit aufdrängen? Anders gefragt: Dient tatsächlich alles, was in Kindertageseinrichtungen einem Beobachter in der Gestalt von Dingen ins Auge fällt, jenen Zielen und Zwecken, die der frühpädagogische Diskurs der Praxis als Intention zuschreibt?

Der Beitrag knüpft an dieser Frage an und stellt die angestammte frühpädagogische Sicht der Dinge ausgehend von Beobachtungen auf die Probe, wie sie sich aus einer „Ethnographie der Frühpädagogik“ (vgl. hierzu Honig und Neumann 2013) gewinnen lassen. Der epistemische Stellenwert einer „Ethnographie der Frühpädagogik“ wird darin gesehen, dass sie an die Stelle des bestehenden disziplinären Wissens über das, was Frühpädagogik vermeintlich auszeichnet, den Blick auf die Selbsterzeugung der frühpädagogischen Praxis im Medium ihrer Vollzugswirklichkeit treten lässt. Ausgangspunkt des Beitrags ist vor diesem Hintergrund die zunächst einmal genauso grundlegende wie triviale Beobachtung, dass in frühpädagogischen Settings noch lange nicht allen Dingen eine auf den ersten Blick erkennbare pädagogische Zwecksetzung inne wohnt. Er rückt damit Sachverhalte in den Blick, die so bislang nicht Gegenstand frühpädagogischer Reflexionen gewesen sind. Die pädagogische Dimension der Dinge wird dabei im Horizont eines Zugangs aufgegriffen, der sich dafür interessiert, wie in frühpädagogischen Settings mit alltäglichen Dingen Pädagogik ,gemacht' wird. Im Mittelpunkt steht 
also nicht die Frage, was an den Dingen pädagogisch ist, sondern wie die Dinge als Dinge zu pädagogischen Dingen werden. Der Beitrag zielt in diesem Sinne darauf ab, die angestammte frühpädagogische Sicht der Dinge in strategischer Weise durch einen verfremdenden Blick zu suspendieren. Er setzt also nicht voraus, immer schon wissen zu können, was mit den Dingen überhaupt geschieht. Vielmehr geht es darum, der frühpädagogischen Vollzugswirklichkeit im Gestus des Entdeckens zu begegnen und das Erkenntnispotential jenes offensiven Verhältnisses zum „Nicht-Wissen“ (Amann und Hirschauer 1997, S. 11) auszuschöpfen, das sowohl für die ,indigene“ Ethnographie der Sozialwissenschaften wie auch die ethnologische und kulturanthropologische Ethnographie charakteristisch ist. Anhand von exemplarischen und explorativen Beobachtungen zum Gebrauch von alltäglichen Gegenständen rekonstruiert der Beitrag dabei, wie diese Gegenstände für die Selbsterzeugung dieser Praxis als einer ,pädagogischen' einen spezifischen Stellenwert gewinnen. Die folgenden Ausführungen versuchen entsprechend einer Pädagogik der Dinge empirisch auf die Spur zu kommen. Sie verorten sich damit im Horizont des Programms einer „Empirie des Pädagogischen“ (vgl. Neumann 2010) und gehen ihm hier anhand von ethnographischen Beobachtungen zum Gebrauch von alltäglichen Gegenständen in Kinderkrippen nach. ${ }^{1}$ Im Mittelpunkt steht die Frage, was man über das Pädagogische der Frühpädagogik erfährt, wenn man sie von der materielldinglichen Dimension ihrer Vollzugswirklichkeit her betrachtet.

\section{2 „Tanz um den Teppich“}

Es ist Mittwochvormittag, die Zeit nach dem Frühstück. Ich befinde mich im Gruppenraum der ,, Goldhamster “, in dem 16 Kinder im Alter zwischen 8 und 48 Monaten betreut werden. Auf dem gelben Linoleumboden in der Nähe der Tür zum Wickelraum liegt ein mehrfarbiger, etwa 5 Quadratmeter grosser Teppich mit arabischen Ziffern an den Rändern. Er hat die Form eines Kreises. Auf dem Teppich befinden sich neben Stofftieren und Spielzeugen auch einige Kinder (9-11 Monate alt). Zwei von ihnen, Lara und Rolf, liegen in der Mitte auf ihren Bäuchen, zwei weitere, Maria und Pierre, in Babywippen, die am Rand des Teppichfeldes platziert sind. Neben Pierre sitzt Marguerite auf einem weit nach unten gefahrenen Bürostuhl. In der Hand hält sie eine Schale mit Brei, in der sie mit einem Plastiklöffel herum rührt und dann damit beginnt, Pierre zu füttern. Ich beobachte immer wieder andere Personen dabei, wie sie sich dem Teppich nähern, dann aber um ihn herum tänzeln, auf seinen Rändern balancieren oder vor ihm plötzlich stoppen, um ihn dann in weiten Schritten auf den Zehenspitzen zu überqueren. Nach einer Weile sehe ich, wie Martine (35 Monate) einen Softball vom gelben Linoleumboden aus auf den Teppich schleudert. Als der Ball auf dem Teppich zum Liegen kommt, läuft sie auf ihn zu und hebt ihn auf. Marguerite, die immer noch auf dem Bürostuhl sitzt und Pierre füttert, kehrt ihren Oberkörper um und ruft in Richtung Martine: „Martine, ihr sollt doch nicht so wild durch die Gegend rennen!". Martine zieht sich mit betont lang(sam)en Schritten vom Teppich auf den Linoleumboden zurück, dreht sich um und beginnt zu grinsen.

Ein teilnehmender Beobachter, der sich in den Räumlichkeiten von Kinderkrippen bewegt, trifft dort unweigerlich auf eine ganze Reihe von nicht-belebten Entitäten, denen man - einerseits - aufgrund ihrer physisch-haptischen Qualität durchaus den Status von 
„Dingen“ zuschreiben kann, die aber - andererseits - auch nicht ohne weiteres bereits in ihrer pädagogischen Bedeutsamkeit unmittelbar hervortreten. Neben Bilderbüchern, Bauklötzen, Wasserfarbkästen, Klettergerüsten und Musikinstrumenten besteht das Inventar von Kinderkrippen eben auch aus Telefonen, Laptops, Fotocollagen, Pinnwänden, Wartesesseln, Bilderrahmen, Fachbüchern, Backöfen, Stühlen und vielem anderem mehr. Hinzu kommt, dass diese Art von Inventar - anders als etwa der Wasserfarbkasten beim Malen oder das Bilderbuch beim Betrachten - in seiner Vorhandenheit zumeist hintergründig bleibt, die Gegenstände in ihrem Dingcharakter also über weite Strecken gar nicht erst „auffallen“, weil von ihnen beim Gebrauch oder im Zuge einer flüchtigen Registratur erst gar keine objektivierende Notiz genommen wird. Im Anschluss an Heidegger könnte man in diesem Zusammenhang präzisierend auch von „Zuhandenem“ sprechen (Heidegger 1927/1979, S. 69-74), das sich vom „Vorhandenen“ insofern unterscheidet, als es - wie etwa der Schraubenzieher beim Schrauben oder die Lampe beim Leuchten - zwar ,zur Hand“ ist, aber in seinem Status als Ding solange unscheinbar bleibt, bis es allein „für sich" betrachtet wird.

Der Teppich, auf dem sich die Ereignisse in der beschriebenen Szene abspielen, gehört ebenfalls zu jener Menge an ,zuhandenem“ Inventar, das sich kaum einmal in seinem Dingcharakter aufdrängt. Spielzeuge und Stofftiere werden auf ihm ablegt und wieder aufgehoben, Babywippen darauf platziert, versetzt und umgedreht, er wird von Reinigungskräften gesaugt, zusammen- und wieder aufgerollt, von eintretenden Besuchern beiläufig wahrgenommen und wieder vergessen. Dennoch gibt das Bewegungsverhalten sowohl der um ihn herum als auch auf ihm selbst befindlichen Personen in dieser mehrminütigen Sequenz zu erkennen, dass er allein durch sein Zuhandensein an der Ordnung des Geschehens in der Kinderkrippe seinen gleichsam „stillschweigenden“ Anteil hat. Der Teppich fungiert in der Aufteilung des nicht weiter durch Wände untergliederten Raums als eine Art ,Nest“, in dem Kinder, die noch nicht in der Lage sind, selbst zu sitzen oder zu laufen, territorial vom übrigen Geschehens abgeschirmt werden. Nimmt der Teppich im Kontext des Geschehens aber die Rolle eines Nestes ein, so lässt sich dies wohl kaum allein mit dem Verweis auf seine blosse Eigenschaft als Bodenbelag erklären. Der Teppich ist nicht lediglich ein Einrichtungsgegenstand, sondern ein Element in der territorialen Ordnung des Gruppenraumes, in dessen Funktion sich auch die Relationen zwischen den Elementen dieser territorialen Ordnung widerspiegeln. Jenseits dieser Relationen, wäre die jeweilige Besonderheit der einzelnen Zonen kaum begreifbar. Genauer: Der Teppich könnte als bewegungsarmer Sonderbezirk für einen Beobachter nicht als eine Art „Nest“ in Erscheinung treten, wenn er nicht an jene Zone grenzen würde, die sich im Verhältnis zu ihm als ein Areal der relativen „Unordnung“ erweist. Dies ist zugleich das Kernmoment seiner ordnungsstiftenden Funktion: Wie jedes ordnungsbildende Moment unterscheidet auch er gleichsam zwischen Ordnung und Unordnung (vgl. hierzu Anter 2007).

Die Ordnung, welche der Teppich territorial stiftet, beruht aber nicht lediglich auf seiner räumlichen Strukturierungsfunktion. Vielmehr wird diese erst dadurch wirksam, dass der Teppich auch die Verhaltensweisen der im Raum befindlichen Personen relationiert. Anders gesagt: Er schafft eine territoriale Ordnung, in die eine soziale Ordnung des Geschehens eingeschrieben ist. Vor allem das sich über mehrere Minuten hinweg erstreckende „Herumtänzeln“ der Erzieherinnen und Kinder signalisiert in dieser Hin- 
sicht, dass sich in seiner Randlage im Raum wie auch seinem gewöhnlichen Gebrauch als Lagerstätte für „,noch-nicht laufende“ Kinder bestimmte Erwartungen an das Verhalten der Akteure materialisieren. Der Umgang mit seiner Platzierung indiziert vor diesem Hintergrund, dass der Ordnung des Geschehens im Gruppenraum eine Unterscheidung zwischen „liegenden“, „sitzenden“ und „laufenden“ Personen zugrunde liegt. Sie ist sozusagen ständig im Spiel, wenn in Kinderkrippen zwischen einzelnen Kindern oder bestimmten Gruppen von Kindern unterschieden wird, so etwa bei ihrer Zuordnung zu bestimmten Räumen, der Planung und Gestaltung des Tagesablaufs oder der Aufteilung des Personals. Sozial bedeutsam ist sie in dieser Situation, weil sie auch hier die wechselseitige Wahrnehmung der Anwesenden und ihres Verhaltens im Umfeld des Teppichs orientiert. Die Platzierung des Teppichs bringt also eine Unterscheidung immer wieder aufs Neue hervor, die ihr bereits als Kalkül zugrunde lag. Im Lichte dessen wirkt die Ermahnung der Erzieherin an Martine wie eine Ausnahme, die eine implizite Regel bestätigt: Laufende Kinder und Erwachsene sollen von dieser Fähigkeit auf dem Teppich selbst einen möglichst unauffälligen Gebrauch machen.

Über diese verhaltensregulierende Funktion hinaus zeigt die Szene aber auch, wie ein so unscheinbares „Ding“ wie jener Teppich im besagten Gruppenraum selbst zu einem Schlüsselelement bei der Pädagogisierung des Geschehens werden kann. Als ordnungsbildendes Moment schränkt er nämlich nicht nur die Kontingenzen des Geschehens im Raum ein, sondern macht auch - wie im Falle von Martine - regelabweichendes Verhalten objektivierbar. Deutlich wird dies, wenn man sich vor Augen führt, dass Marguerites Reaktion auf Martines Verhalten weder als notwendig noch als zufällig angesehen werden kann. Als Martine dem auf den Teppich geschleuderten Ball hinterherläuft, wird dies von Marguerite als eine Störung des regelkonformen Betriebsablaufs thematisiert, ohne dass dies durch die Situation selbst zwingend vorgegeben wäre. Vielmehr wäre auch anderes möglich gewesen, etwa die Deutung von Martines Verhalten als ein Ausdruck selbstgesteuerter motorischer Lernaktivitäten oder als eine Form der bildungsbedeutsamen Aneignung der Bewegungs- und Gravitationseigenschaften eines Balls. Marguerites Intervention löst dabei den Teppich aus seiner stillschweigenden Zuhandenheit heraus und lässt gerade damit Martines Verhalten als abweichend hervortreten. Als Martine langsamen Schrittes vom Teppich zurückweicht, wirkt der Teppich wiederum als eine Art Resonanzboden, welcher der nachgängigen Korrektur ihres Verhaltens Ausdruck verleiht.

In der Einheit von Intervention und performativer Verhaltenskorrektur spiegelt sich wie in einer Miniatur die pädagogische Relevanz der Situation. Sie besteht darin, dass sich der Vollzug der Verhaltenskorrektur unmittelbar mit der Repräsentation der Wirksamkeit des Vollzogenen verknüpft. Im ethnomethodologischen Sinne könnte man entsprechend vom Pädagogischen als einer Form der darstellenden Herstellung gerade stattfindender Personenveränderung sprechen, die es - als eine Form der sinnvermittelnden Wirklichkeitserzeugung - der pädagogischen Praxis erst ermöglicht, sich selbst als pädagogisch zu beobachten (vgl. hierzu Neumann 2012b, S. 233 ff.). Die pädagogische Reflexionstradition bringt diesen Umstand nachgerade unwissentlich zur Sprache, wenn sie sich auf Erziehung, Bildung oder Lernen zugleich als Ziel wie Mittel intendierter Personenveränderung bezieht (vgl. Oelkers und Tenorth 1991). Vor diesem Hintergrund ermöglicht es der Teppich, Kinder als buchstäblich in Veränderung begriffene Personen zu objektivieren, als solche nämlich, die noch nicht sind, was sie einmal werden, aber 
schon anders sein können, als diejenigen, die sie gerade eben noch sind. Sie treten dabei nicht lediglich als objektanaloge Adressen pädagogischer Interventionen in Erscheinung, sondern - ähnlich wie der Teppich selbst - als Kollaborateure, die entscheidend an der Pädagogisierung des Geschehens beteiligt sind (vgl. hierzu auch Jung 2009).

Möglich ist dies alles jedoch nur, weil es offenkundig so etwas wie ein „konjunktives“ Erfahrungswissen (vgl. Mannheim 1980) darüber gibt, wie man sich im Einzugsbereich des Teppichs zu bewegen hat. Dies wiederum unterstreicht zugleich seine Bedeutung als ein effektives Moment im Kontext des ,hidden curriculum“ der Kinderkrippe, die nicht einfach Kinder beaufsichtigt oder gar bildet, wie es die frühpädagogische Sicht der Dinge verheißt (vgl. hierzu Liegle 2010), sondern auch in bestimmte, die institutionelle Ordnung erst herstellende Routinen hinein sozialisiert. Ganz offensichtlich transzendiert der Teppich dabei seinen gewöhnlichen Status als Einrichtungsgegenstand bzw. Bodenbelag und übernimmt die Funktion eines Schutzraums, dessen Effektivität als physisch gar nicht wirklich abschirmender Raumteiler darin besteht, wie der geräuschlose Dirigent eines sozialräumlichen Ensembles zu wirken, der - um mit Bruno Latour zu sprechen „Vorbeikommende“ dazu zwingt, „Rollen in seiner Erzählung zu spielen“ (Latour 2006, S. 485). In diesem Sinne „kommuniziert“ er gleichsam Verhaltenserwartungen, die von den beteiligten Personen scheinbar nur mehr orchestriert zu werden brauchen. Mit der Entfaltung seiner sozialen Brisanz verliert der Teppich jedoch zugleich jede substanzielle Qualität, ist weder länger in sich eingegrenzt noch äußerlich abgeschlossen, weswegen seine Bezeichnung als ein „Ding“ im Grunde genommen auch irreführend ist. Vielmehr operiert er als ein Artefakt, dessen situative Artifizialität ihm nicht als ein von Menschenhand gefertigtes „Ding“ bereits zukommt, sondern erst im Umgang mit ihm entsteht und auch von diesem Umgang stets abhängig bleibt. Ein Teppich beginnt zwar auch in Kinderkrippen seine Karriere in der Regel als Bodenbelag. Jedoch muss es dabei nicht bleiben. Insofern verweisen die Beobachtungen zum Umgang mit dem Teppich zugleich auf die Grenzen eines naiv-dingontologisch präformierten Blicks. Genauso wie der Teppich seine Eigenschaften erst durch seine relationale Einbettung in das Geschehen und nicht etwa durch sich selbst als Teppich erlangt, ist seine Funktion keineswegs ein für alle Mal festgeschrieben. Dies könnte nicht zuletzt Anlass bieten, seiner Statuskarriere im Horizont einer „Biographie der Dinge“ (Doering und Hirschauer 1997) nachzuspüren. Im Grunde gilt dies aber für alle vermeintlich nicht-pädagogischen Dinge, die in den Räumen von Kinderkrippen hausen. Entsprechend ist es auch wenig verwunderlich, wenn man in Kinderkrippen regelmäßig auf seine funktionalen Äquivalente trifft, die als Bodenmatten, Wolldecken oder Schaffelle das Gleiche wie der Teppich in der beschriebenen Szene bewirken.

\section{3 ,Wozu sind Schuhe da?““}

Ottokar (27 Monate) bewegt sich zielstrebig von der Stirnseite des etwa 25 Quadratmeter grossen Gruppenraumes Richtung Puppenecke. In der Mitte des Raumes angekommen, schlendert er kurz, tritt etwas schief und ich beobachte, wie er dabei beide Schuhe verliert. Ottokar läuft trotzdem munter weiter, bis er die Puppenecke erreicht hat. Danach kehrt er um und peilt sofort die gegenüberliegende Seite des Raums an. Dabei stolpert 
er fast über seine verlorenen Schuhe. Als er sie entdeckt, hebt er sie auf und katapultiert sie mit einem gezielten Wurf außerhalb der von ihm angepeilten „Laufstrecke“. Danach trippelt er barfüssig weiter auf das gegenüberliegende Ende des Raumes zu. Trixie, die etwa zwei Meter daneben steht, ruft Ottokar zu: „Nicht mit den Schlappen!“. Ihre Kollegin Annemarie ergänzt: ,, Schuhe sind für die Füße!". Schließlich macht Trixie einige Schritte auf Ottokar zu und fragt rhetorisch: ,, Wozu sind die Schlappen da?". Ottokar bleibt stumm und blickt sie nur an, während sie ihn unter den Armen packt, hoch hebt und ihn schliesslich vor den verlorenen Schuhen wieder absetzt. Danach bückt sie sich und zieht ihm die Schuhe wieder an. ,, So, jetzt lauf weiter! “.

Der Anblick einer schieren Unmenge von Schuhen gehört regelmäßig zu den sowohl flüchtigsten wie auch prägnantesten Eindrücken, die man beim Betreten einer Kindertageseinrichtung gewinnen kann. Wenn man die Garderobe passiert, stiftet er stets einen kurzen Moment des Staunens, das kurz darauf der wiederkehrenden Gewissheit weicht, es bei der öffentlichen Kinderbetreuung mit einer Massenveranstaltung zu tun zu haben. Trotz seiner Flüchtigkeit entfaltet der Anblick dabei einen durchaus beachtlichen Informationswert: An der Menge der Schuhe auf den unteren Regalleisten der Garderoben kann man beispielsweise nicht nur ablesen, ,wieviel heute los ist“ oder ob man eher „früh“ oder „spät“ dran ist, man erhält auch das eindeutige Signal, dass es hier alles andere als üblich ist, die Räumlichkeiten hinter der Garderobe mit Schuhen zu betreten. An der Größe der Schuhe wird dabei ebenso offensichtlich, dass diese Regel zumindest für die Kinder ohne Ausnahme gilt.

In der beschriebenen Szene geht es jedoch um eine andere Art von Schuhen als diejenigen, die in den Garderoben gelagert werden. Im native code der beiden Erzieherinnen ist von „Schlappen“ die Rede. Dem designierten Verwendungszweck nach unterscheiden sich diese Schlappen jedoch nicht von den Schuhen im Allgemeinen, denn auch sie stellen eine Art von Fussbekleidung dar. Als solche sind sie allerdings bestimmten Räumlichkeiten vorbehalten, deren Schleusen sich erst nach Durchschreiten einer vielerorts als Eingangsbereich bezeichneten Zone öffnen. Trägt man - auch als Ethnograph - die sogenannten „Schlappen“, so ist dies nicht zuletzt ein untrügliches Zeichen dafür, dass man eine gewisse Grenze überschritten und eine Zutrittserlaubnis zu den Gruppenräumen erlangt hat. Im Sinne Goffmans (2009) unterscheiden sich diese Gruppenräume von der eher komplexen Form der Öffentlichkeit des Eingangsbereichs insofern, als in ihnen die Anonymität von Beobachtungen und unterstellten Beobachtungserwartungen von der Erfahrung abgelöst wird, dass hier tatsächlich jeder jeden beobachtet. Es ist nicht zuletzt der Topos des „Schlappen-Tragens“, der dies auf eindeutige Weise indiziert, was freilich weniger an den „Schlappen“" selbst liegt, als daran, dass man damit rechnen muss, auf sie angesprochen oder zumindest mit Schildern und Symbolen auf sie hingewiesen zu werden, sollte man sie einmal nicht tragen. Damit steigt schließlich in erheblichem Maße die Wahrscheinlichkeit, dass es zu Situationen kommt, in denen die „Schlappen“ den Status der Zuhandenheit als „Schuhwerk“ verlassen - mithin: als „Dinge“ relevant werden.

Genau dies passiert in der geschilderten Szene. In ihr wiederholt sich insofern auf gleichsam archetypische Weise jener Vorgang simultaner „Pädagogisierung und Verdinglichung“, dem auch der Teppich in der vorherigen Sequenz seine ausgezeichnete Funktion als Mittler verhaltensregulierender wie verhaltenskorrigierender Maßnahmen verdankt. Die Szene zeigt vor diesem Hintergrund, dass die „Schlappen“ zunächst ein- 
mal bei den Beteiligten keine besondere Aufmerksamkeit erregen, solange sie sich an Ottokars Füßen befinden und sich ihm nicht als ein Hindernis in den Weg stellen. Auch als Ottokar sie aufhebt und anschließend beiseite schleudert, scheint er sich ihrer Zuhandenheit noch gewiss zu sein. Der Verlust der Schuhe selbst stört ihn jedenfalls nicht. Das Gleiche trifft auf die anwesenden Erzieherinnen zu. Auch deren Reaktion gilt nicht dem Umstand, dass Ottokar an seinen Füssen keine Schuhe mehr trägt. Vielmehr richtet sie sich auf ein offenkundig nicht regelkonformes Verhalten, das von ihnen jedoch nicht explizit als ein Verstoß, sondern als ein unsachgemäßer Gebrauch gekennzeichnet wird: Mit den Schlappen soll nicht geworfen werden, denn sie sind schließlich „,ür die Füße!“”. Damit aber transzendieren Ottokars verlorene Schuhe den unscheinbaren Status der bloßen ,Zuhandenheit" und werden zu einer verdinglichten Materialität. Sie sind dabei nicht einfach mehr ,zur Hand“, sondern werden nun auch in ihrer singulären Objekthaftigkeit als ein vorhandenes ,Solches“ thematisiert.

Bemerkenswert daran ist zunächst einmal, dass es in dieser Situation offenbar darauf ankommt, wie Ottokar mit den „Schlappen“ umgeht, und nicht etwa darauf, ob er sie noch am Fuß trägt. Es ist also nicht der Verlust des Schlappens, der zur Intervention veranlasst und es geht offenbar auch nicht darum, dass sich Ottokar die „Schlappen“ selbst wieder anziehen soll. Im Vordergrund steht vielmehr die Mahnung an ihren bestimmungsgemäßen Gebrauch: „Wozu sind die Schlappen da?“. In diese rhetorisch zu verstehende Frage der Erzieherin Trixie ist eine Fähigkeitsadressierung eingeschrieben, die einer Subjektivierung (vgl. hierzu Reh und Ricken 2012) gleichkommt und mit einer Responsabilisierung einhergeht: Sie unterstellt, dass Ottokar wissen kann, wie die „Schlappen“ zu benutzen sind. Bezeichnend ist in diesem Zusammenhang, dass die Antwort von Annemarie kurz zuvor bereits vorweg genommen wurde und auch Trixie eine mögliche Antwort Ottokars gar nicht erst abwartet, sondern sie gleichsam selbst unmittelbar herbeiführt. Dies wiederum unterstreicht nochmals den rhetorischen Charakter ihrer Frage. Verlangt ist von Ottokar nicht eine Information, sondern seine ,Zustimmung“ zum bestimmungsgemäßen Gebrauch der „Schlappen“. Die Zustimmung wird jedoch nicht einfach der Entscheidung Ottokars anheimgestellt, sondern durch den Zugriff auf den Körper des Kindes und das darauf folgende Anziehen der Schuhe ganz gezielt erbracht.

Die Sequenzialität der Situation offenbart gewisse Eigentümlichkeiten des pädagogischen Umgangs mit den Dingen, die auf die bereits am Beispiel zuvor rekonstruierte Verknüpfung von Intervention und Verhaltenskorrektur verweisen. Die buchstäbliche „Auseinandersetzung“ (Cassirer 1985, S. 55) von Ding und Person, durch die beide letztlich erst als einander Gegenüberstehende hervortreten, eröffnet dabei zunächst einmal überhaupt erst den Raum für eine Pädagogisierung der Situation. Dies zeigt sich schon daran, dass die kommunikative Signifizierung der Schuhe und die verhaltensevaluative Ansprache ihres Trägers sich nachgerade simultan vollziehen. Anders gesagt: Die Objektivierung des „Schlappens“ als Vorhandenheit ist mit der „Subjektivierung“ seines Trägers unmittelbar verknüpft. Verdinglichung auf der einen und Personalisierung auf der anderen Seite vollziehen sich also ko-extensiv. Ähnlich wie der implizite Verweis auf die ungeschriebenen Gesetze für das Bewegungsverhalten im Umfeld des Teppichs in der zuvor beschriebenen Situation regelabweichendes Verhalten und seine Korrektur zur Aufführung bringt, ermöglicht es in dieser Szene die Objektvierung der Schuhe, ihren Träger als zu verändernde Person in Erscheinung treten zu lassen. 
Eine solche Finalisierung des Geschehens liegt keineswegs auf der Hand: Genauso gut hätte man sich etwa vorstellen können, dass die Erwachsenen lediglich ihrer Sorge um eine Beeinträchtigung von Ottokars Wohlbefinden bei einer möglichen Erkältung nachgeben und ihm die „Schlappen“ einfach kommentarlos wieder anziehen. Dies ist jedoch nicht der Fall. Vielmehr wird die Situation so „gelenkt“", dass dabei ein möglicher Lerneffekt herauskommen oder mindestens doch als solcher provoziert werden kann. In ihr manifestiert sich so ein in der frühpädagogischen Praxis empirisch immer wieder zu beobachtendes Muster: In die Adressierung von Kindern als Personen ist immer auch die Konstitution dieser Personen als gleichermaßen veränderungsbedürftig wie veränderungsfähig eingeschrieben (vgl. hierzu etwa Neumann 2013; Schulz 2013). Die Intervention der Erzieherinnen gewinnt im Zuge dessen am Ende einen performativen Charakter: Sie stellt her, was als mögliche Wirkung des Eingreifens vorausgesetzt wird. Anders gesagt: Fähigkeitsadressierung und Befähigung gehen Hand in Hand. Man hat es also hier nicht nur mit einer Praxis zu tun, die eine Personenveränderung zu bewirken verspricht, sondern im Akt des Vollzugs immer auch schon ihre personenverändernde Wirksamkeit zur Darstellung bringt. Das Pädagogische des Geschehens besteht somit gleichsam in einer Art Technik, die genau dies ermöglicht, nämlich die Ebene der Herstellung sich verändernder Personen mit der Ebene der Darstellung der Wirksamkeit dieser Veränderung unmittelbar zu verknüpfen. Allerdings wäre die Performativität des Geschehens nicht denkbar, ohne dass sich neben den professionellen Akteuren auch die Kinder am pädagogischen Spiel beteiligen. Dinge nehmen bei der sozialen Organisation dieses Vorgangs eine Schlüsselrolle ein als gleichsam stille, aber wirkmächtige Teilhaber. Einerseits sind sie am objektivierenden Vollzug der spezifischen Unterscheidung von Kindern als Kindern beteiligt, andererseits profitieren sie aber auch genau davon wiederum in ihrem epistemologischen Status als Dinge.

Im Hinblick auf die Empirie einer Pädagogik der Dinge ist diese Analyse äußerst aufschlussreich. Einerseits zeigt sie nämlich, dass die pädagogische Dimension der Dinge nicht auf Eigenschaften beruht, die ihnen gleichsam substanziell gegeben sind.

Vielmehr gewinnen sie sowohl ihre Eigenschaften als Dinge als auch ihre pädagogische Bedeutsamkeit erst im Zuge einer bestimmten Art und Weise des verdinglichenden Umgangs mit ihnen als materiellen Gegenständen. Das bedeutet aber wiederum: Es gibt kein Ding, das von sich aus bereits pädagogisch oder pädagogisch bedeutsamer wäre als ein anderes, aber auch keines, mit dem sich nicht so etwas wie Pädagogik machen ließe.

Vorgänge der Verdinglichung lassen sich in diesem Kontext so zugleich lesen als Ereignisse der pädagogischen Sinnkonstitution, die gerade in der sozialen Verwobenheit wechselseitiger Objektivierungsverhältnisse auf die transintentionale Finalität des pädagogischen Geschehens verweisen.

\section{Resümee: Das Pädagogische der Frühpädagogik im Spiegel ihrer Materialität}

Die pädagogische Dimension der Dinge ist in jüngster Zeit verstärkt in den Horizont der erziehungswissenschaftlichen Aufmerksamkeit gerückt. Noch markiert dies nicht mehr als einen Trend. Allerdings dürfte die Frage nach der paradigmatischen Bedeutung dieses Trends auch eher zweitrangig sein, ist sie doch vor allem für die Chronisten und 
Programmatiker der Disziplin und weniger für die Forschung selbst von Interesse. Durchaus berechtigt ist aber die Frage, worin sein epistemischer Stellenwert besteht. Anders gesagt: Es geht darum, ob sich dieser alternative Zugang zum Gegenstand „Pädagogik“ auch mit einem Zuwachs an Wissen über diesen Gegenstand verknüpft.

In dieser Hinsicht liebäugelt die erziehungswissenschaftliche Forschung mit dem Versprechen, dass der Blick auf die Dinge mehr und anderes sieht, weil er der Komplexität der Erziehungswirklichkeit eher gerecht wird als etwa eine Perspektive, die nur von der unmittelbaren Aufdringlichkeit des zwischenmenschlichen Geschehen ausgeht (vgl. etwa Asbrand et al. in diesem Heft). Seine Pointe besteht dann in nichts Geringerem als darin, die in der Entwicklung der Erziehungswissenschaft selbst kaum einmal problematisierte Selbstverständlichkeit zu überwinden, pädagogische Phänomene ausschließlich in der Einheit von Person, Zeit und Handlung zu denken. Der Erfolg klassischer Theorien über das Pädagogische der Erziehungswirklichkeit, wie etwa derjenigen vom ,,pädagogischen Bezug“ (Nohl 1935), beruht nicht zuletzt auf diesem Naturalismus. Mit der Perspektive auf die dinglich-materielle Realität der Erziehungswirklichkeit jedoch wird das Pädagogische nicht mehr länger an humane Handlungsträger und ihre Absichten oder bestimmte Orte und Situationen gebunden. Vielmehr wird es gerade in seiner prinzipiell verstreuten Sozialität zum Thema.

Vor allem mit Blick auf die Frühpädagogik kann ein solcher Perspektivwechsel ein gehöriges Irritationspotential entfalten. Ihre bildungstheoretischen Visionen sind nämlich durchgängig davon geprägt, dass sie die Dinge zu „Miterziehern“ erklärt (vgl. Stieve 2008, S. 42), die eine kaum zu ignorierende „Aufforderung zur Bildung“ kommunizieren (Liegle 2009, S. 11). Entsprechend wahllos geht die Frühpädagogik damit um, den Dingen eine pädagogische Bedeutung zuzuschreiben. Letztlich kann sie dann gar nicht anders als alles, was in frühpädagogischen Settings zwischen Menschen und Dingen geschieht, immer schon als Bildungsprozess zu betrachten. Diese Lesart unterstellt jedoch, die Komplexität und Multireferentialität der Wirklichkeit frühpädagogischer Institutionen ließe sich in der vorgesellschaftlichen Idylle der Auseinandersetzung des einzelnen Kindes mit sich und seiner materiellen Umwelt auflösen. Die Frühpädagogik bekräftigt damit schließlich den Kindheitsbegriff der bürgerlichen Moderne: Er konzipiert das Kind als ein natürliches und ganzheitliches Wesen, das noch nicht zivilisiert ist und sich in einer noch ganz vorsozialen Welt bewegt (vgl. Richter 1987; Baader 1996).

Die Beobachtungen zum Umgang mit den vermeintlich nicht-pädagogischen Dingen verdeutlichen nicht zuletzt vor diesem Hintergrund, wie weit das Pädagogische der Frühpädagogik davon entfernt ist, lediglich mit dem identisch zu sein, was der fachliche Diskurs unter Bildung versteht. Insofern gestattet es der empirische Blick auf die Materialität der Frühpädagogik nicht nur, Sachverhalte zu thematisieren, die ihr pädagogisches Vorurteil bislang nicht gesehen hat, sondern auch, vermeintlich bekannte Sachverhalte auf bislang unvorhergesehene Weise zu betrachten. Phänomene wie Lernen, Bildung oder Entwicklung lassen sich so nämlich als Hervorbringungen einer sozialen Praxis studieren, die im Umgang mit den Dingen erst erzeugt, was sie voraussetzen muss, um die von ihr antizipierten Resultate überhaupt am einzelnen Kind festmachen zu können. Dies ermöglicht wiederum, die vermeintlich bereits beantwortete Frage nach ,dem Pädagogischen“ der Frühpädagogik empirisch neu zu stellen. Anders gesagt: Es handelt sich um eine Blickschneise der Gegenstandsexploration, die sowohl auf eine veränderte 
Gegenstandsauffassung vorausweist wie auch die Grenzen der gängigen frühpädagogischen Gegenstandsauffassung zur Sprache bringen kann.

Aber auch in diesem Perspektivwechsel verbirgt sich noch eine naturalistische Versuchung. Sie besteht darin, sich der Anschaulichkeit der Dinge nicht entziehen zu können und so womöglich das (Überraschungs-)Potential einer Dezentrierung des frühpädagogischen Blicks der ungezügelten ,Lust am Konkreten` zu opfern. Die vorhergehenden Ausführungen hinterlassen vor diesem Hintergrund die Botschaft, dass es gerade mit Blick auf den epistemischen Status der Dinge als Dinge irreführend sein kann, immer schon von ihnen als einer für sich bestehenden, präoperativen Tatsache auszugehen. Insofern kann es nicht allein darum gehen, die Subjekt/Objekt-Trennung einerseits zu kritisieren (vgl. Latour 2002), sie aber auch anderseits immer schon vorauszusetzen, um sie dann letztlich in einer virtuosen Beschreibung verschiedenster Mensch-Ding-Assoziationen wieder ontologisch zu relativieren (vgl. Kneer 2008). Vielmehr sollte es gerade auch um die Frage gehen, wie diese Trennung vollzugslogisch überhaupt erst einmal zustande kommt und welche Funktion dieses Zustandekommen für eine Praxis hat, die von sich selbst als einer besonderen Praxis ausgeht. Zur Debatte steht also die Frage nach jenen verdinglichenden Weisen des Umgangs mit den Dingen, welche die frühpädagogische Praxis sowohl ermöglichen wie auch in ihren Möglichkeiten begrenzen.

\section{Anmerkung}

1 Zurückgegriffen wird dabei auf Ergebnisse und Analysen des ethnographischen Forschungsprojekts „Betreuungswirklichkeit und Bildungswirklichkeit. Die Pädagogik der Maison Relais pour Enfants“, das im Zeitraum 2009-2012 an der Universität Luxemburg durchgeführt und mit Mitteln des Forschungsfonds der Universität sowie des luxemburgischen Ministeriums für Familie und Integration finanziert wurde. Die teilnehmende Beobachtung im Rahmen des Projektes umfasste insgesamt 6 Einrichtungen, die in mehreren, bis zu 8-wöchigen Feldphasen von bis zu 3 Forschern untersucht worden sind. Ausgewählt wurden sie auf der Basis eines snowball sampling, das neben den Kriterien der Trägerschaft und der Regionalität auch die Größe der Einrichtungen sowie die Alterszusammensetzung der Kindergruppen berücksichtigte. Die jeweiligen Erhebungen stützten sich auf unterschiedliche Verdatungsstrategien und Datenformate, zu denen neben Beobachtungsprotokollen, Video- und Fotoaufnahmen sowie Notizen in Feldtagebüchern auch Artefakte und Dokumente gehörten.

\section{Literatur}

Amann, K., \& Hirschauer, S. (1997). Die Befremdung der eigenen Kultur. Ein Programm. In S. Hirschauer \& K. Amann (Hrsg.), Die Befremdung der eigenen Kultur. Zur ethnographischen Herausforderung soziologischer Empirie (S. 7-52). Frankfurt a. M.: Suhrkamp.

Anter, A. (2007). Die Macht der Ordnung. Aspekte einer Grundkategorie des Politischen (2., überarbeitete Auflage). Tübingen: Mohr Siebeck.

Baader, M. S. (1996). Die romantische Idee des Kindes und der Kindheit. Auf der Suche nach der verlorenen Unschuld. Neuwied: Luchterhand. 
Bollig, S., Kelle, H., \& Seehaus, R. (2012). (Erziehungs-)objekte beim Kinderarzt. Zur Materialität von Erziehung in Kindervorsorgeuntersuchungen. Zeitschrift für Pädagogik, 58. Beiheft, 218-237.

Breidenstein, G. (2006). Teilnahme am Unterricht. Ethnographische Studien zum Schülerjob. Wiesbaden: VS Verlag für Sozialwissenschaften.

Casale, R., König, G. M., \& Priem, K. (Hrsg.). (2012). Die Materialität der Erziehung: Kulturelle und soziale Aspekte pädagogischer Objekte. Weinheim: Beltz.

Cassirer, E. (1985). Form und Technik. In E. Cassirer (Hrsg.), Symbol, Technik, Sprache (Aufsätze 1927-1933) (S. 39-91). Hamburg: Meiner.

Doering, H., \& Hirschauer, S. (1997). Die Biographie der Dinge. Eine Ethnographie musealer Repräsentation. In S. Hirschauer \& K. Amann (Hrsg.), Die Befremdung der eigenen Kultur. Zur ethnographischen Herausforderung soziologischer Empirie (S. 267-297). Frankfurt a. M.: Suhrkamp.

Eßer, F. (2012). Assembling the Resident Child. Membership in Residential Care. In M.-S. Honig \& S. Neumann (Hrsg.), (Doing) Ethnography in Early Childhood Education and Care (S. 133151). Luxembourg: Université du Luxembourg.

Fetzer, M. (2012). Lernen in einer Welt der Dinge. Methodologische Diskussion eines Objekt-integrierenden Ansatzes zur mikroethnographischen Unterrichtsanalyse. In B. Friebertshäuser, H. Kelle, H. Boller, S. Bollig, C. Huf, A. Langer, M. Ott, \& S. Richter (Hrsg.), Feld und Theorie. Herausforderungen erziehungswissenschaftlicher Ethnographie (S. 121-135). Opladen: Barbara Budrich.

Fritzsche, B., Idel, T.-S., \& Rabenstein, K. (2011). Ordnungsbildung in pädagogischen Praktiken. Praxistheoretische Überlegungen zur Konstitution und Beobachtung von Lernkulturen. Zeitschrift für Soziologie der Erziehung und Sozialisation, 31, 28-44.

Goffman, E. (2009). Interaktion im öffentlichen Raum. Frankfurt a. M.: Campus.

Heidegger, M. (1927/1979). Sein und Zeit (15., durchgesehene Auflage). Tübingen: Niemeyer.

Hewitt, K. (2001). Blocks as a Tool for Learning: Historical and Contemporary Perspectives. Young Children, 56, 6-13.

Honig, M.-S., \& Neumann, S. (2013). Ethnographie der Frühpädagogik. Einführung in den Themenschwerpunkt. Zeitschrift für Soziologie der Erziehung und Sozialisation, 33, 4-9.

Jung, P. (2009). Kindertageseinrichtungen zwischen pädagogischer Ordnung und den Ordnungen der Kinder: Eine ethnographische Studie zur pädagogischen Reorganisation der Kindheit. Wiesbaden: VS Verlag für Sozialwissenschaften.

Kneer, G. (2008). Hybridizität, zirkulierende Referenz, Amoderne? Eine Kritik an Bruno Latours Soziologie der Assoziationen. In G. Kneer, M. Schroer, \& E. Schüttpelz (Hrsg.), Bruno Latours Kollektive. Kontroversen zur Entgrenzung des Sozialen (S. 261-305). Frankfurt a. M.: Suhrkamp.

König, G. M. (2012). Das Veto der Dinge. Zur Analyse materieller Kultur. Zeitschrift für Pädagogik, 58. Beiheft, 14-31.

Langeveld, M. J. (1955). Das Ding in der Welt des Kindes. Zeitschrift für Pädagogik, 1, 69-83.

Latour, B. (2002). Die Hoffnung der Pandora. Frankfurt a. M.: Suhrkamp.

Latour, B. (2006). Über technische Vermittlung. Philosophie, Soziologie, Genealogie. In A. Belliger \& D. Krieger (Hrsg.), ANThology. Einführendes Handbuch zur Akteur-Netzwerk-Theorie (S. 483-528). Bielefeld: transcript.

Latour, B. (2007). Eine neue Soziologie für eine neue Gesellschaft. Einführung in die Akteur-Netzwerk-Theorie. Frankfurt a. M.: Suhrkamp.

Liegle, L. (2009). Wir brauchen eine Didaktik der indirekten Erziehung. Betrifft Kinder 6(9), 6-13.

Liegle, L. (2010). Didaktik der indirekten Erziehung. In G. E. Schäfer, R. Staege, \& K. Meiners (Hrsg.), Kinderwelten - Bildungswelten. Unterwegs zur Frühpädagogik (S.11-25). Berlin: Cornelsen Scriptor.

Mannheim, K. (1980). Strukturen des Denkens. Frankfurt a. M.: Suhrkamp. 
Meyer-Drawe, K. (1999). Herausforderung durch die Dinge. Das Andere im Bildungsprozess. Zeitschrift für Pädagogik, 45, 329-342.

Mollenhauer, K. (1987). Die Dinge und die Bildung. In: K.-D. Braun \& D. Wunder (Hrsg.), Neue Bildung - Neue Schule. Wolfgang Klafki zum sechzigsten Geburtstag (S. 32-46). Weinheim: Beltz.

Neumann, S. (Hrsg.). (2010). Beobachtungen des Pädagogischen. Programm - Methodologie Empirie. Luxembourg: Université du Luxembourg.

Neumann, S. (2012a). Pädagogisierung und Verdinglichung. Beobachtungen zur Materialität der Frühpädagogik. Zeitschrift für Pädagogik, 58. Beiheft, 168-184.

Neumann, S. (2012b). Beobachtungsverhältnisse. Feldtheoretische Erkundungen zu einer Empirie des Pädagogischen. In S. Bernhard \& C. Schmitt-Wellenburg (Hrsg.), Feldanalyse als Forschungsprogramm (S. 221-242). Wiesbaden: VS Verlag für Sozialwissenschaften.

Neumann, S. (2013, i.E.). Die sozialen Bedingungen der Bildung. Beobachtungen im Feld der Frühpädagogik. In G. Breidenstein, K. Jergus, \& C.Thompson (Hrsg.), Interferenzen. Perspektiven kulturwissenschaftlicher Bildungsforschung. Weilerswist: Velbrück Wissenschaft.

Nohl, A.-M. (2011). Pädagogik der Dinge. Bad Heilbrunn: Klinkhardt.

Nohl, H. (1935). Die pädagogische Bewegung in Deutschland und ihre Theorie. Frankfurt a. M.: Schulte-Bulmke.

Oelkers, J. (2012). Die Historizität pädagogischer Gegenstände. Zeitschrift für Pädagogik, 58. Beiheft, 32-49.

Oelkers, J., \& Tenorth, H.-E. (Hrsg.). (1991). Pädagogisches Wissen. Weinheim: Beltz.

Parmentier, M. (2001). Der Bildungswert der Dinge oder: Die Chance des Museums. Zeitschrift für Erziehungswissenschaft, 4, 39-50.

Pazzini, K.-J. (1983). Die gegenständliche Umwelt als Erziehungsmoment. Zur Funktion alltäglicher Gebrauchsgegenstände. Weinheim: Beltz.

Prochner, L. (2011). ,Their little wooden bricks': A History of the Material Culture of Kindergarten in the United States.

Rabenstein, K., \& Wieneke, J. (2012). Der Blick auf die Dinge des Lernens. Überlegungen zur Beobachtung der materiellen Dimension pädagogischer Praktiken. In H. de Boer \& S. Reh (Hrsg.), Beobachtung in der Schule - Lernen Beobachten (S.189-202). Wiesbaden: VS Verlag für Sozialwissenschaften.

Reh, S., \& Ricken, N. (2012). Das Konzept der Adressierung. Zur Methodologie einer qualitativempirischen Erforschung von Subjektivation. In I. Miethe \& H.-R. Müller (Hrsg.), Qualitative Bildungsforschung und Bildungstheorie (S. 35-56). Opladen: Barbara Budrich.

Richter, D. (1987). Das fremde Kind. Zur Entstehung der Kindheitsbilder des bürgerlichen Zeitalters. Frankfurt a. M.: Fischer.

Rieger-Ladich, M. (2009). Menschen und Dinge, Akteure und Aktanten. Überlegungen zur Neubestimmung des Sozialen. In B. Grubenmann \& J. Oelkers (Hrsg.), Das Soziale in der Pädagogik. Zürcher Festgabe für Reinhard Fatke (S. 114-130). Bad Heilbrunn: Klinkhardt.

Röhl, T. (2012). From Witnessing to Recording - Material Objects and the Epistemic Configuration of Science Classes.

Rousseau, J. J. (1971). Emile oder über die Erziehung. Paderborn: Ferdinand Schöningh.

Schatzki, T. R. (1996). Social practices. A Wittgensteinian Approach to Human Activity and the Social.

Schatzki, T. R. (2001). The Site of the Social. A Philosophical Account of the Constitution of Social Life and Change.

Schulz, M. (2013). Frühpädagogische Konstituierung von kindlichen Bildungs- und Lernprozessen. Zeitschrift für Soziologie der Erziehung und Sozialisation, 33, 26-41.

Sørensen, E. (2009). The Materiality of Learning: Technology and Knowledge in Educational Practice. 
Stieve, C. (2008). Von den Dingen lernen. Die Gegenstände unserer Kindheit. Paderborn: Wilhelm Fink.

Stieve, C. (2011). Inszenierte Dingwelten. Spiel- und Lernmaterialien in Kindertageseinrichtungen. In P. Cloos \& M. Schulz (Hrsg.), Kindliches Tun beobachten und dokumentieren. Perspektiven auf die Bildungsbegleitung in Kindertageseinrichtungen (S. 115-129). Weinheim: Juventa.

Wiesemann, J. (2009). ,Handwerk des Lernens‘. Zum kulturellen Selbstverständnis schulischen Lernens im Sachunterricht. In H. Giest, R. Lauterbach, \& B. Marquart-Mau (Hrsg.), Lernen und kindliche Entwicklung - Elementarbildung und Sachunterricht (S.269-276). Bad Heilbrunn: Klinkhardt. 